\title{
cGMP-dependent protein kinase $1 \beta$ phosphorylates and regulates the function of the actin/myosin-associated protein caldesmon
}

\author{
Darren E Casteel", Raphaela Schwappacher, Hema Rangaswami, Jacqueline Su-Yuo \\ From 6th International Conference on cGMP: Generators, Effectors and Therapeutic Implications \\ Erfurt, Germany. 28-30 June 2013
}

\section{Background}

The type I cGMP-dependent protein kinases (PKGI $\alpha$ and PKGI $\beta$ ) are splice variants that differ in their first $\sim 100$ amino acids, giving each isoform unique dimerization and autoinhibitory domains. The unique coiled-coil dimerization domains mediate isoform specific proteinprotein interactions, and we have previously identified the amino acids that are important in mediating the interaction between PKGI $\beta$ and its two known interaction partners, TFII-I and IRAG [1].

\section{Results}

Using wild-type and mutant PKGI $\beta$ D/D domains as affinity probes in a proteomic screen and we identified the actin/myosin associated protein caldesmon as a PKGI $\beta$ specific interacting protein [2]. Using immunofluorescent staining, we found that PKGI $\beta$ and $\mathrm{CaD}$ colocalized with $\mathrm{F}$-actin at lammellipodial structures at the edge of MDAMB-231 cells. We found that PKGI phosphorylated $\mathrm{CaD}$ in a species- and isoform-specific manner. Human type 5 caldesmon was phosphorylated on serine 12 by PKGI $\beta$ in vitro and in intact cells. Phosphorylation on serine 12 or a phospho-mimetic S12E mutation significantly reduced the interaction between $\mathrm{CaD}$ and myosin IIA. We found that siRNA mediated caldesmon depletion increases the migration of MDA-MB-231 cells, and that reconstitution with wild-type or phospho-deficient S12A caldesmon slowed migration. In contrast, migration was not slowed by reconstitution with caldesmon containing an S12E mutation. We also found that PKG activation leads to indirect phosphorylation of mouse and human $\mathrm{CaD}$ in $293 \mathrm{~T}$ and MDA-MB-231 cells. The indirect phosphorylation seen in 293T cells is accompanied by a shift in the apparent molecular mass of $\mathrm{CaD}$ during SDS-PAGE. The observed migratory shift is similar to that previously seen when purified platelet $\mathrm{CaD}$ was directly phosphorylated in vitro by PKA [3]. Indirect phosphorylation of $\mathrm{CaD}$ in MDAMB-231 did not cause a migratory shift.

\section{Conclusion}

Since serine 12 is not conserved in mouse or rat, our results indicate that PKGI $\beta$ regulates caldesmon in a species-specific manner. While the PKGI-mediated indirect phosphorylation site(s) have not been determined, our preliminary data suggests that PKGI could potentially regulate all $\mathrm{CaD}$ isoforms, albeit in a cell type and species specific manner.

\section{Published: 29 August 2013}

\section{References}

1. Casteel DE, Boss GR, Pilz RB: Identification of the interface between cGMP-dependent protein kinase Ibeta and its interaction partners TFII-I and IRAG reveals a common interaction motif. J Biol Chem 2005, 280:38211-38218.

2. Schwappacher R, Rangaswami H, Su-Yuo J, Hassad A, Spitler R, Casteel DE: cGMP-dependent protein kinase Ibeta regulates breast cancer cell migration and invasion via a novel interaction with the Actin/Myosinassociated protein caldesmon. J Cell Sci 2013, 126:1626-1636.

3. Hettasch JM, Sellers JR: Caldesmon phosphorylation in intact human platelets by CAMP-dependent protein kinase and protein kinase C. J Biol Chem 1991, 266:11876-11881.

doi:10.1186/2050-6511-14-S1-P13

Cite this article as: Casteel et al:: cGMP-dependent protein kinase $\mathbb{I}$ phosphorylates and regulates the function of the actin/myosinassociated protein caldesmon. BMC Pharmacology and Toxicology 2013 14(Suppl 1):P13.

\footnotetext{
* Correspondence: dcasteel@ucsd.edu

Department of Medicine and Cancer Center, San Diego, La Jolla, California 92093, USA
}

(c) 2013 Casteel et al; licensee BioMed Central Ltd. This is an Open Access article distributed under the terms of the Creative Commons Attribution License (http://creativecommons.org/licenses/by/2.0), which permits unrestricted use, distribution, and reproduction in any medium, provided the original work is properly cited. 